\title{
Coworking como modalidade e espaço de trabalho: uma análise bibliométrica
}

\author{
Paloma Fraga Medina ${ }^{1}$, Edite Krawulski ${ }^{2}$ \\ Universidade Federal de Santa Catarina (Florianópolis, SC, Brasil)
}

Coworking é a modalidade de trabalho na qual profissionais de diferentes áreas sem local fixo de trabalho buscam ampliar sua rede de contatos, compartilhando espaço e serviços de escritório. Considerando essa realidade, o artigo apresenta resultados de análise bibliométrica sobre o tema coworking, a qual objetivou identificar estudos e seus enfoques no trato da temática. Foram analisados oito artigos, identificados por meio de busca sistematizada em bases de dados, a qual possibilitou identificar as áreas do conhecimento que vêm estudando o coworking, a metodologia de pesquisa utilizada, os períodos das publicações e seus países de origem. Os resultados foram categorizados conforme a abordagem relacionada ao tema. Os artigos identificados foram produzidos na França, Inglaterra, Estados Unidos, Austrália, Alemanha e Espanha, e publicados majoritariamente em 2012, sendo, em sua maioria, oriundos da área de informática. Predominaram estudos de abordagem qualitativa, associando o tema coworking a relações de trabalho, a novas formas de trabalho, ao espaço físico, ao perfil dos coworkers e à responsabilidade social.

Palavras-chave: Coworking, Novas formas de trabalho, Bibliometria, Espaço de coworking.

Coworking as working mode and space: A bibliometric analysis

Coworking is the working mode in which professionals from different areas without fixed workplace seek to expand their networking by sharing the same space and business services. Considering this fact, the article presents results of a bibliometric analysis on the coworking subject which aimed to identify studies and their approaches in the treatment of this theme. Eight articles were analyzed, identified through systematic search in databases, which enabled us to identify the areas of knowledge that have been studying coworking, research methodology used, periods of publications, and the countries of origin of the studies. Results were divided into categories according to the related approach to the subject. The articles identified were produced in France, England, USA, Australia, Germany, and Spain, and were published mainly in 2012, coming mostly from the computer science area. Studies conducted through qualitative approach predominated, associating the theme coworking to labor relations, new ways of working, physical space, profile of coworkers, and social responsibility.

Keywords: Coworking, New ways of working, Bibliometry, Coworking space.

\section{Introdução}

A s transformações ocorridas nos processos produtivos nas duas últimas décadas do século XX devido ao desenvolvimento acelerado da tecnologia, ao capitalismo e à globalização, entre outros fatores, reformularam o lugar do homem no trabalho, assim como sua forma de atuar e os espaços utilizados para realização das atividades (Antunes, \& Alves, 2004). O debate sobre as mudanças na sociedade e nos contextos produtivos, e suas implicações nas relações sociais, particularmente quanto ao modo como as pessoas se articulam nessas relações na contemporaneidade, tem permeado alguns estudos nas últimas décadas (Antunes, \& Alves, 2004; Bauman, 2004; Chanlat, 1996; Grisci, Scalco, \& Kruter, 2011). Esses estudos, em sua maioria, convergem em apontar a precariedade e a instabilidade das atuais condições e relações de trabalho e as consequências dessas mudanças na vida profissional e pessoal dos trabalhadores.

O crescimento do denominado trabalho flexível é característico do século XXI, visto que a internet e o desenvolvimento tecnológico possibilitam a realização das atividades laborais independentemente do lugar em que o sujeito se encontra (Eaton, 2001). Para Aquino

1 Mestre em Psicologia na área de Psicologia das Organizações e do Trabalho na Universidade Federal de Santa Catarina.

2 Professora Associada do Departamento de Psicologia da Universidade Federal de Santa Catarina. 
(2007) a flexibilidade, muitas vezes, é estratégica, permitindo e justificando a sobrevivência da organização produtiva e comparecendo como um dos principais elementos que caracteriza, na contemporaneidade, o vínculo entre tempo e trabalho. Na concepção de Bauman (2001), a flexibilidade parte de uma perspectiva segundo a qual o trabalho flexível representa o fim da relação com o emprego tradicional e sua substituição por contratos de curto prazo (ou sem contratos), posições sem cobertura previdenciária e muitas incertezas sobre o futuro do trabalhador, colocando-o em uma posição vulnerável.

As transformações tecnológicas estão no cerne do trabalho flexível, uma vez que não se apresentam apenas em equipamentos ou processos, mas também em produtos e na dinâmica das empresas (Bianchetti, 2001) por meio da informalização do trabalho, visto o aumento de terceirizados, subcontratados, trabalhadores em tempo parcial, ou ainda, teletrabalhadores (Antunes, 2012). Nesse cenário, com suas múltiplas modalidades possíveis de exercício da atividade produtiva, vêm emergindo, em escala mundial, os escritórios de coworking, onde atuam profissionais denominados coworkers. Em tradução livre coworker significa "colega de trabalho" ou "trabalhar conjuntamente". No contexto deste artigo, este termo nomeia o trabalhador que atua em coworking, isto é, em uma modalidade sob a qual profissionais de diferentes áreas, em busca de enriquecer sua rede de contatos e/ou por não possuírem um local fixo de trabalho, compartilham o mesmo ambiente e serviços de escritório (Costa, 2013a).

Considerando a dificuldade em encontrar registros que evidenciem a verdadeira história da gênese do coworking, Jackson (2013), em dissertação sobre o futuro dos espaços de trabalho, traçou um breve histórico a partir de suas investigações. De acordo com a autora, o termo coworking foi utilizado pela primeira vez por Brad Neuberg em 2005 para descrever um espaço de trabalho compartilhado por alguns freelancers ${ }^{3}$ em São Francisco, nos Estados Unidos, e denominado de "Coworking Group". O coworking funcionava durante três dias da semana em um centro de saúde, o Spiral Muse, no período em que não ocorriam as atividades regulares do espaço, sendo que nos demais dias Brad e seus colegas buscavam outros lugares para trabalhar. Cerca de um ano depois, diante das limitações do Spiral Muse, as pessoas que ali atuavam criaram o coworking Hat Factory. Porém, quando o Hat Factory encerrou suas atividades, após algum tempo, uma nova parceria surgiu entre Brad Neuberg, Tara Hunt e Chris Messina, criando um novo espaço de coworking, o Citizen Space, para disseminar pelo mundo essa nova modalidade de trabalho.

O coworking ainda é fenômeno recente no Brasil: os primeiros movimentos de implementação dessa iniciativa em nosso país datam de 2007, mostrando-se como tendência cada vez maior no mercado de trabalho atual. Desde então, o coworking divulga entre suas vantagens o baixo custo e o fornecimento de uma estrutura adequada para atender pequenas empresas, autônomos, freelancers, empresários emergentes e teletrabalhadores.

De acordo com dados de uma pesquisa alemã feita pela Deskmag (Cashman, 2012), o Brasil ocupa a sétima posição mundial em número de coworkings por país. O líder do top 10 da pesquisa ainda são os EUA, berço do coworking, sendo que a Espanha domina o ranking em relação a novos espaços de coworking abertos. Dentre as propostas centrais do modelo de coworking estão: possibilitar um espaço de trabalho para profissionais que estão fora da estrutura tradicional de organização, mas buscando também permitir que o isolamento profissional seja reduzido ao estimular o compartilhamento e ofertar o networking.

3 O freelancer exerce uma forma independente de trabalho, com maior autonomia e flexibilidade, se comparado a profissionais regulamentados em organizações. Tem o isolamento como fator inerente ao seu trabalho, mas ao mesmo tempo estende seus contatos a uma diversidade maior de pessoas. Os pequenos empresários, por vezes, são vistos como semelhantes ao perfil de freelancer. Entretanto, seu contato é muito mais estreito, devido ao número baixo de funcionários e à escala de negócios atendidos (Uda, 2013). 
Pesquisa realizada por Costa (2013b) para o blog Movebla ${ }^{4}$, com abrangência apenas no Brasil, evidenciou que o maior número de coworkers em nosso país se caracteriza por empreendedores. Já dados da Deskmag (Cashman, 2012) indicam que 53\% dos coworkers são freelancers, enquanto os demais são empresários ou funcionários de alguma empresa. A "atmosfera social agradável" foi considerada ainda, segundo a Deskmag, como um dos principais motivos para a escolha pelo coworking como modalidade/ambiente de trabalho. Em segundo lugar, dentre esses motivos, ficou a "comunidade" e em terceiro a "interação com os outros". Percebe-se, então, certa necessidade de contato com outros profissionais, seja pelo bom clima que o coworking possa oferecer ou pelo aspecto de comunidade e de troca de favores e ideias, o que permeia é a necessidade de interagir, o que não seria tão acessível caso os trabalhadores optassem pelo home office ou por outros espaços possíveis de trabalho, como livrarias, cafés, bibliotecas etc.

A busca por trabalho sob diferentes arranjos e modalidades, como, por exemplo, em coworking, pode ser decorrência da exaustão de modelos tradicionais de trabalho, como também pode derivar de oportunidades que se vão configurando a partir de novas demandas, ou ainda, de razões não suficientemente conhecidas pelos estudiosos. Considerando a relevância de investigar a respeito desses novos arranjos, este artigo apresenta uma análise bibliométrica das publicações científicas sobre coworking, realizada com o intuito de conhecer o direcionamento dos estudos efetuados e as tendências para apoiar futuras pesquisas com essa temática. Para tanto, foram identificados os estudos publicados sobre o tema coworking, quais áreas publicaram sobre o assunto, a quantidade de artigos publicados por ano e em quais países esse tema tem sido objeto de estudo.

\section{Método}

Para o desenvolvimento deste estudo, primeiramente, utilizou-se o Portal Capes ${ }^{5}$, e através do ícone "Acervo" optou-se por "todas as áreas do conhecimento" para então definir as bases de busca. Das 259 bases sugeridas pelo Portal, apenas sete forneceram artigos relacionados a coworking com o enfoque proposto para esta pesquisa: Scopus, Chemoreception Abstracts, Compendex Engineering Index, Emerald, Highwire Press, Science Direct e Springerlink. Não foi estabelecida restrição de país ou período de publicação para a busca efetuada, tendo em vista seu caráter exploratório e o objetivo de analisar de forma ampla as contribuições identificadas sobre o tema no contexto geral.

Optou-se por iniciar a pesquisa pela base Scopus, pois ela indexa conteúdo científico de múltiplas áreas. Com os resultados obtidos foram definidas as chaves de busca nas versões em português e inglês: "coworking", "novas formas de trabalho" e "espaço de coworking". Estabeleceuse ainda que para fazer parte do escopo em análise os artigos deveriam tratar do tema coworking como modalidade de trabalho ou como espaço compartilhado de trabalho.

A leitura sistematizada dos títulos e resumos, realizada na sequência, possibilitou excluir os artigos que não atendiam aos requisitos estabelecidos e, ainda, levantar informações sobre o quantitativo de publicações com o tema coworking, as áreas de pesquisa a respeito, os tipos de pesquisa utilizados, as datas das publicações e os países de origem dos estudos desenvolvidos sobre este tema. Como último procedimento, os achados da pesquisa foram subdivididos em categorias de análise, conforme a abordagem relacionada ao tema coworking.

4 Disponível em: <www.movebla.com >.

5 http://www.periodicos.capes.gov.br/ 


\section{Apresentação e discussão dos resultados}

A busca em diferentes bases de dados gerou como resultado a identificação de 540 publicações, das quais 502 foram excluídas após leitura dos títulos e dos resumos, em virtude de não representarem temas de fato associados ao coworking, como o proposto para esta pesquisa. Visto que o termo coworking pode significar "trabalhar em conjunto", muitos artigos abordam o termo associando-o a relações de trabalho em geral. Dos 38 estudos restantes, 21 eram duplicados, restando, dessa forma, apenas 17 artigos, dos quais se realizou leitura na íntegra. Essa leitura conduziu, ainda, à exclusão de nove desses artigos por não tratarem em específico sobre coworking como modalidade ou como espaço compartilhado de trabalho, mas sim abordando o tema sob o enfoque do compartilhamento de aprendizagem em bibliotecas públicas, como terminologia para outras situações, ou, ainda, como novas configurações de trabalho não relacionadas ao coworking. Assim, para a amostra final deste estudo foram selecionados oito artigos (Figura 1).

Figura 1. Fluxograma de escolha dos estudos

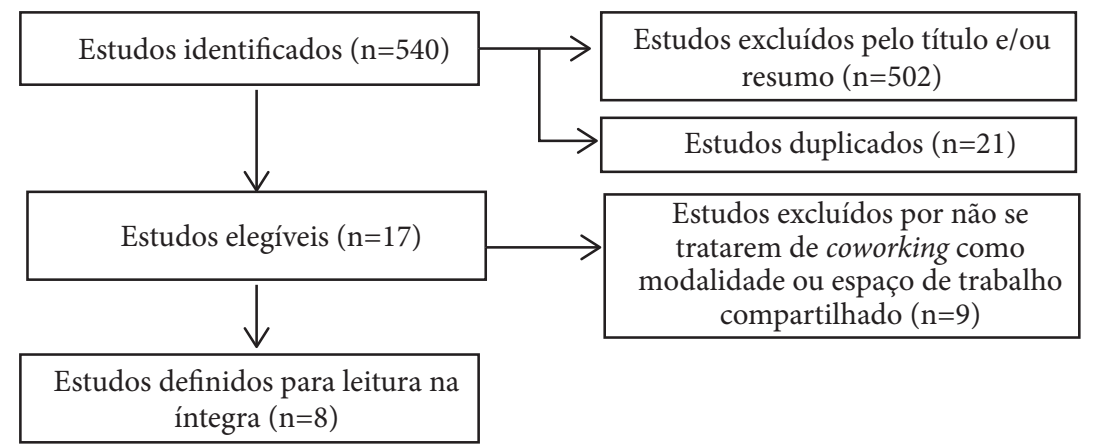

As produções científicas selecionadas foram publicadas entre 2008 e 2015, com maior concentração em 2012. O menor número de publicações a respeito do tema foi em 2008 (apenas um artigo). É importante destacar também que, referente ao ano de 2015, foram analisados artigos publicados até o mês de abril (Tabela 1).

Tabela 1. Caracterização dos resultados

\begin{tabular}{|c|c|c|}
\hline Características & & \\
\hline Ano & Número de artigos & $\%$ \\
\hline 2008 & & 12,5 \\
\hline 2012 & $\begin{array}{l}1 \\
3\end{array}$ & 37,5 \\
\hline 2013 & 2 & 25 \\
\hline 2015 & 2 & 25 \\
\hline Países & Número de artigos & $\%$ \\
\hline Austrália & 2 & 25 \\
\hline Alemanha & 2 & 25 \\
\hline Espanha & 1 & 12,5 \\
\hline Estados Unidos & 1 & 12,5 \\
\hline França & 1 & 12,5 \\
\hline Inglaterra & 1 & 12,5 \\
\hline Áreas de Pesquisa & Número de artigos & $\%$ \\
\hline Tecnologia da Informação & 5 & 62,5 \\
\hline Ciências Sociais & 2 & 25 \\
\hline Engenharia & 1 & 12,5 \\
\hline
\end{tabular}


A pesquisa resultou em artigos originários da Austrália, Alemanha, Espanha, Estados Unidos, França e Inglaterra, porém nenhum registro de estudo produzido no Brasil. Essa ausência de publicações brasileiras sobre a temática certamente se relaciona ao fato de a modalidade de coworking ser ainda muito recente no Brasil: os primeiros escritórios compartilhados neste formato emergiram em 2007 e, a partir de então, esta nova configuração de trabalho têm se disseminado em nosso país. De acordo com dados do último Censo Coworking Brasil (Costa, 2015), no ano de 2007 havia apenas sete coworkings, enquanto o ápice de novos espaços abertos se deu em 2014, com 56 coworkings. Até março de 2015, segundo a mesma fonte, foram contabilizados mais 10, os quais, somados àqueles abertos nos demais anos, totalizam 238 espaços de coworking no Brasil. Embora esses quantitativos indiquem que estamos diante de um fenômeno em crescimento na realidade ocupacional brasileira, percebe-se que ele ainda não foi objeto de produção científica sistematizada e publicada, uma vez que não foram encontrados registros de artigos sobre o assunto originados no nosso país, como visto na Tabela 1.

Quanto ao delineamento metodológico das produções identificadas, todas são estudos qualitativos, sendo 66,67\% estudos empíricos e, 33,33\%, teóricos. Já em relação às áreas temáticas, a maior parte dos artigos origina-se da Tecnologia da Informação $(62,50 \%)$ e, em menor frequência, das Ciências Sociais (25\%) e Engenharia (12,50\%). Pesquisa realizada pela Deskmag (Foertsch, 2011) mostrou que a maioria dos cowokers era de profissionais da área de tecnologia, desenvolvedores ou programadores; talvez esses dados possam explicar o maior número de artigos escritos sobre o tema na área de Tecnologia da Informação.

A análise a respeito dos enfoques sob os quais o coworking vem sendo estudado nessas diferentes áreas indicou que ele contempla cinco grandes temas: a) Relações de trabalho (Aguiton, \& Cardon, 2008; Andrade, Ares, Suárez, \& Giret, 2013; Bilandzic, Schroeter, \& Foth, 2013), no que tange às relações no ambiente de coworking ou a formas facilitadoras de relações nesses espaços; b) Novas formas de trabalho (Brinks, 2012; Pohler, 2012), tratando sobre as diferentes configurações de trabalho; c) Espaço de trabalho (Kojo, \& Nenonen, 2014), abordando o modo como os espaços se organizam em seu aspecto físico e suas influências no desenvolvimento do trabalho e sua caracterização; d) Perfil dos coworkers (Spinuzzi, 2012), buscando caracterizar o perfil dos trabalhadores desses espaços, suas expectativas e a forma como o percebem como nova modalidade de trabalho; e e) Responsabilidade social (Mouat, \& Buksh, 2015), referindo-se a questões sustentáveis relativas ao compartilhamento. Cada um desses temas será tratado em pormenor na sequência (Tabela 2).

\begin{tabular}{|c|c|c|c|}
\hline Títulos & Autores & Países & Categorias \\
\hline $\begin{array}{l}\text { Implementation challenges for supporting coworking } \\
\text { virtual enterprises }\end{array}$ & $\begin{array}{l}\text { Andradde, Ares, } \\
\text { Suarez \& Giret }\end{array}$ & Espanha & Relações de trabalho \\
\hline $\begin{array}{l}\text { Gelatine: making coworking places gel for better } \\
\text { collaboration and social learning }\end{array}$ & $\begin{array}{c}\text { Bilandzic, } \\
\text { Schroeter \& Foth }\end{array}$ & Austrália & Relações de trabalho \\
\hline $\begin{array}{l}\text { Bercamps and coworking: Collective web-based } \\
\text { infrastructures for innovation }\end{array}$ & Aguiton \& Cardon & França & Relações de trabalho \\
\hline $\begin{array}{l}\text { Networking practices and social gatherign in Coworking } \\
\text { Spaces - Working between digitalization and relocalization }\end{array}$ & Brinks & Alemanha & $\begin{array}{l}\text { Novas formas de } \\
\text { trabalho }\end{array}$ \\
\hline $\begin{array}{l}\text { Neue Arbeitsräume für neue Arbeitsformen: Coworking } \\
\text { Pohler Spaces }\end{array}$ & Pohler & Alemanha & $\begin{array}{l}\text { Novas formas de } \\
\text { trabalho }\end{array}$ \\
\hline $\begin{array}{l}\text { Places for multi-location work - opportunities for facilities } \\
\text { management }\end{array}$ & Kojo \& Nenonen & Inglaterra & Espaço de trabalho \\
\hline $\begin{array}{l}\text { Workig Alone Together: Coworking as Emergent } \\
\text { Collaborative Activity }\end{array}$ & Spinuzzi & Estados Unidos & Perfil dos coworkers \\
\hline $\begin{array}{l}\text { Activating smart wor hubs for urban revitalisation: } \\
\text { evidence and implications of digital urbanism for planning } \\
\text { and policy from South-East Queensland }\end{array}$ & Mouat \& Buksh & Austrália & Responsabilidade social \\
\hline
\end{tabular}




\section{Relações de trabalho}

Entre os estudos analisados, os artigos de Andrade et al. (2013) e de Bilandzic et al. (2013) tratam de ferramentas virtuais que buscam ampliar as relações de trabalho entre coworkers do mesmo ou de diferentes coworkings, como forma de estímulo ao compartilhamento e à troca de ideias e informações. Salientam os citados autores que um dos objetivos principais desta modalidade de trabalho, ao menos em sua concepção, é exatamente o estímulo às interações sociais.

Ainda sobre as relações de trabalho, Aguiton e Cardon (2008) discorrem sobre os barcamps, conhecidos como "desconferência", ou seja, uma conferência sem programação. Trata-se de uma rede que se forma com o modelo de conferência, na qual profissionais apresentam seus projetos e, através da interação com os participantes, buscam aperfeiçoá-lo. O artigo defende que a vontade de colaborar é o principal fator nestas relações; seja em barcamps ou no próprio coworking, o objetivo daqueles que se agregam a esse tipo de iniciativa é estabelecer redes de contatos e colaborar abertamente e de maneira horizontal com os demais profissionais.

De fato, a ideia de colaboração e compartilhamento é um dos objetivos centrais do coworking. Sua estrutura física e de gestão se propõe a buscar, a todo momento, o estímulo das relações interprofissionais, oferecendo ambientes abertos sem interferência de paredes ou de quaisquer outros obstáculos visuais, assim como a promoção de workshops entre os coworkers, como recurso para apresentarem seus trabalhos e habilidades e ampliarem suas redes de contatos. Parece paradoxal que com todas as ferramentas tecnológicas que buscam facilitar a comunicação, a sociedade contemporânea ainda vivencie a solidão, a qual emerge, segundo Grisci, Bitencourt e Fleck (2012), a partir das exigências por melhores desempenhos no trabalho, assim como da cobrança de inovação para a inclusão no mercado de trabalho em uma sociedade que parece primar pela competitividade. Dessa forma, seja por meio de ferramentas virtuais, como Andrade et al. (2013) e Bilandzic et al. (2013) sugerem, ou mesmo por meio dos barcamps (Aguiton, \& Cardon, 2008), que permitem o contato pessoal, desenvolver melhores formas de estímulo ao networking entre os coworkers tem sido apontada como tarefa dos gestores de coworking.

Muitos coworkers buscam essa modalidade de trabalho com o objetivo de ampliarem suas redes de contato, assim como de interagirem mais e sentirem-se mais criativos. Em consonância com os discursos vigentes na atualidade, esses trabalhadores têm reconhecido o impacto e a importância da temática das relações de trabalho nos contextos laborais, e, para tanto, procuram possibilidades de estabelecer o networking. $\mathrm{O}$ enfoque nessas relações poderia, porém, além de facilitar os contatos interpessoais, estimular também a solidariedade e a colaboração, componentes que poderiam até mesmo modificar os sentidos (nem sempre positivos) atribuídos ao trabalho nos dias atuais.

\section{Novas formas de trabalho}

Brinks (2012) lança um olhar sobre o coworking considerando-o como um novo formato de trabalho, com enfoque nos profissionais autônomos que se utilizam desse espaço como forma de ampliação de sua rede de contatos. Espaços de coworking são descritos, assim, como "lugares de mercado" em que o networking é possível em função do próprio ambiente físico que estimula o compartilhamento, entendendo-se que esse ambiente seja uma nova forma de contextualização das situações de trabalho.

Pohler (2012), por sua vez, procura mostrar o aumento do trabalho atípico e não assalariado que vem ocorrendo na Alemanha. Segundo a autora, os novos acordos de trabalho são caracterizados por mais liberdade, flexibilidade e autonomia, e, nesse contexto, os espaços de coworking podem 
ser vistos como uma manifestação de novos regimes de trabalho, assim como as novas formas pelas quais as pessoas percebem o trabalho.

Sabe-se que os avanços tecnológicos contribuem de modo decisivo para essa realidade, na medida em que possibilitam a flexibilização do trabalho e da comunicação, e favorecem a mobilidade dos trabalhadores, permitindo-lhes novos modos de exercer suas atividades laborais. No contexto brasileiro, especificamente, os primórdios do processo de reestruturação produtiva emergiram na década de 1980, quando, ao findar a ditadura militar, inaugurou-se um período no qual muitas empresas passaram paulatinamente a adotar novos padrões organizacionais e tecnológicos, novas formas de organização social do trabalho, bem como programas de qualidade total e sistemas just in time (Antunes, 2012). Num ritmo muito mais lento do que aquele vivenciado em países centrais, obviamente, uma vez que o processo de industrialização é recente, no nosso país têm começado a emergir, igualmente, arranjos laborais caracterizados por maior liberdade, flexibilidade e autonomia por parte dos trabalhadores, e a disseminação de coworking em nosso meio parece confirmar essa realidade.

\section{Espaço de trabalho}

O coworking tem como proposta ser um modelo diferente daquele tradicional de trabalho. De posse dessa ideia, Kojo e Nenonen (2014) abordam a temática sob a ótica do espaço físico, realizando uma revisão da literatura a respeito dos espaços de trabalho desde os anos 1960 até os atuais modelos de trabalho flexível. Os autores buscam ainda identificar os conceitos de serviço e solução de design do local de trabalho pelos quais os objetivos do coworking podem ser satisfeitos. A prestação de serviços organizacionais e escritórios flexíveis, segundo sua concepção, procuram favorecer a socialização dos usuários, garantindo funcionalidades tais como conectividade virtual eficiente, além de acessibilidade.

De acordo com Castells e Cardoso (2005), nosso mundo está em um processo multidimensional de transformação, associado à emergência de um novo paradigma tecnológico, baseado nas tecnologias de comunicação e informação, que se difundiram de forma desigual por todo o mundo. Segundo os mesmos autores, a sociedade é que dá forma à tecnologia, de acordo com as necessidades, valores e interesses de quem a utiliza; o conhecimento e a informação sempre foram centrais na nossa sociedade, mas é novo "o fato de serem de base microeletrônica, através de redes tecnológicas que fornecem novas capacidades a uma velha forma de organização social: as redes" (2005, p.17). Frente às estratégias de formação de redes, a organização ganhou mais flexibilidade com as consequências já referidas, e os espaços de coworking conquistam então terreno fértil para se estabelecerem e angariarem adeptos.

\section{Perfil dos coworkers}

Spinuzzi (2012), a partir de estudo realizado com coworking em Austin (EUA), no qual foram entrevistados seus gestores e coworkers, aborda o perfil dos coworkers e o modo como eles definem o coworking. Refere este autor que a opção pelo trabalho em coworking se deve às dificuldades de trabalhar em casa ou cafeterias, como, por exemplo, as distrações de um ambiente tumultuado, no caso das cafeterias, ou mesmo o isolamento de uma residência, inerentes ao teletrabalho, passível de ser desenvolvido nestes locais. E conclui que o coworking vai muito além de se configurar apenas como um espaço físico compartilhado, caracterizando-se como um lugar onde os profissionais podem criar novas redes e interagir com as mais variadas áreas. 
A questão do perfil de coworkers investigada por Spinuzzi (2012) nos remete à reflexão sobre as significativas mudanças em curso no próprio perfil dos trabalhadores, que de maneira geral procuram por maior formação escolar, atendendo aos apelos por qualificação que lhes são imputados, como também se direcionam com frequência para iniciativas como empreendedores ou empresários. Principalmente nos grandes centros urbanos, esses trabalhadores também não dispensam itens como internet rápida e aparelhos celulares de modelos avançados para realizar suas atividades laborais. Assim, ambos os perfis se aproximam, e a iniciativa do coworking novamente encontra terreno fértil para sua disseminação.

Ainda sabe-se pouco sobre o coworker no contexto nacional brasileiro como um todo, principalmente em regiões e contextos com características socioeconômicas e culturais distintas dos grandes centros urbanos. Mesmo assim, é possível formar uma ideia inicial a respeito do tipo de trabalhador que se dispõe a desenvolver suas atividades profissionais nesse tipo de iniciativa organizacional, seja pela necessidade de enriquecer contatos, seja pela impossibilidade de inserção laboral nos moldes tradicionais.

\section{Responsabilidade social}

Mouat e Buksh (2015) trazem o olhar da responsabilidade social quanto à sustentabilidade, e defendem que espaços como o de coworking facilitam estas questões. Segundo esses autores, a ideia de compartilhamento de espaço por si só torna-se sustentável, visto que o consumo de energia elétrica é reduzido com a ocupação do mesmo ambiente. A redução do consumo de combustível em transportes para o deslocamento até o trabalho é outro fator que tem se destacado, já que a escolha pelo coworking vem pautada em sua localização, sendo notória a preferência, por parte dos coworkers, por aqueles situados mais próximos à sua residência, facilitando o deslocamento a pé ou por meio de bicicleta. Percebe-se que essa concepção, associando o coworking à responsabilidade social, se aproxima, também, dos anseios por maior qualidade de vida no trabalho, muito embora essa aproximação careça de investigação a respeito.

Ao debate sobre essas questões pode ser relacionado o teletrabalho, levando em conta que esse recurso já tem se mostrado como tendência nos últimos anos, sendo apontado como solução para o trabalhador estar próximo à família, evitar trânsito e otimizar seu tempo, bem como conciliar afazeres profissionais com atividades domésticas. Segundo pesquisa, 64\% das empresas brasileiras já permitem que alguns funcionários de determinados cargos trabalhem em casa, mesmo que esporadicamente; mas apenas $11 \%$ delas possuem uma política fixa que possibilita essa forma de trabalho a todos os funcionários (Half, 2012). Parece incontestável que o compartilhamento de espaço e a proximidade casa-trabalho resultem em maior sustentabilidade, na medida em que efetivamente há redução no consumo de energia elétrica e também na queima de combustível, já que boa parte dos profissionais se desloca a pé ou por meio de bicicleta, gerando um modus vivendi socialmente mais responsável.

\section{Considerações finais}

As mudanças decorrentes das constantes transformações tecnológicas demandaram às organizações e aos trabalhadores a necessidade de repensar e reformular seus modos de trabalho. Os novos arranjos organizacionais extrapolam os muros e barreiras que existiam nas empresas tradicionais para se refazerem em contextos mais dinâmicos, criativos e complexos. Nesse contexto, o coworking emerge como uma alternativa para buscar atender às demandas atuais, tanto das 
organizações quanto dos profissionais, por meio de sua configuração estrutural e sua proposta de espaço ocupacional.

Ainda são recentes as contribuições oriundas de estudos sobre coworking, apesar do seu crescimento acelerado nos últimos anos. Considera-se que as publicações analisadas mostraram maior preocupação quanto às formas de gestão e de facilitação do networking, aos incentivos à melhor comunicação, a questões físicas relativas ao espaço de trabalho, ou, ainda, ao modo como se organizam essas novas configurações de trabalho. Sem dúvida, em se tratando de uma temática nova, esses aspectos são relevantes e seu desvelamento contribui para a compreensão do coworking enquanto modalidade e espaço compartilhado de trabalho. Faz-se necessário, no entanto, investigar mais detidamente a respeito do que motiva os profissionais a alterarem suas trajetórias laborais e a buscarem essa modalidade e/ou espaço compartilhado, frente à precarização do trabalho. Pouco se sabe, por exemplo, acerca das implicações dessas mudanças tanto para os trabalhadores quanto para os contextos ocupacionais.

Finalizando, registra-se que a análise apresentada revelou existirem limitações no trato do tema, havendo necessidade de ampliar o escopo de pesquisa a respeito com teses e dissertações, aqui não contempladas. O fato de não terem sido encontradas publicações científicas no contexto brasileiro sobre essa temática, apesar de pesquisas terem mostrado a disseminação de coworking pelo Brasil e pelo mundo, revela, igualmente, uma lacuna que confirma e reforça a necessidade de pesquisas aprofundarem quais são as características dessa modalidade de trabalho na cultura brasileira, como os profissionais a percebem e quem são esses trabalhadores, assim como outras questões que podem emergir na medida em que se adentra na realidade do coworking no Brasil.

\section{Referências}

Aguiton, C., \& Cardon, D. (2008). Barcamps and coworking: Collective web-based infrastructures for innovation. Hermès, 50 (1), 77-82.

Andrade, J., Ares, J., Suárez, S., \& Giret, A. (2013). Implementation challenges for supporting coworking virtual enterprises. Paper presented at the 2013 IEEE 10th International Conference on e-Business Engineering (ICEBE), Coventry.

Antunes, R. (2012). A nova morfologia do trabalho no Brasil: reestruturação e precariedade. Nueva Sociedad (especial em português), 232 (4), 44-59.

Antunes, R., \& Alves, G. (2004). As mutações no mundo do trabalho na era da mundialização do capital. Educação $\mathfrak{E}$ Sociedade, 25 (87), 335-351.

Aquino, C. A. B. (2007). Transformações no modelo industrial, "novos" trabalhos e nova temporalidade. Psicologia $\mathfrak{E}$ Sociedade (edição especial), 19 (1), 21-28.

Bauman, Z. (2004). Amor líquido: sobre a fragilidade dos laços humanos. Rio de Janeiro: Zahar.

Bauman, Z. (2001). Modernidade líquida. Rio de Janeiro: Zahar.

Bianchetti, L. (2001). Da chave de fenda ao laptop - tecnologia digital e novas qualificações: desafios à educação. Petrópolis, RJ: Vozes.

Bilandzic, M., Schroeter, R., \& Foth, M. (2013). Gelatine: making coworking places gel for better collaboration and social learning. Paper presented at the Proceedings of the 25th Australian Computer-Human Interaction Conference: Augmentation, Application, Innovation, Collaboration. Adelaide, Australia.

Brinks, V. (2012). Networking practices and social gathering in Coworking Spaces: working between digitization and re-localization. Geographische Zeitschrift, 100 (3), 129-145.

Cashman, A. (2012). 2000 Coworking Spaces worldwide. Deskmag. Recuperado de https://goo.gl/2C5Oyi [07 novembro 2012] 
Castells, M., \& Cardoso, G. (Org.), (2005). A sociedade em rede: do conhecimento à acção política. Lisboa: Imprensa Nacional.

Chanlat, J.-F. (1996). Quais carreiras e para qual sociedade? (II). Revista de Administração de Empresas, 36 (1), 13-20.

Costa, A. (2013a). Infográfico sobre coworking e a pesquisa brasileira. Recuperado de https://goo.gl/MGboNH [29 maio 2014]

Costa, A. (2013b). Pesquisa coworking no Brasil 2013: os primeiros resultados. Recuperado de https://goo.gl/XCSX1S [27 março 2013]

Costa, A. (2015). Censo coworking: uma breve análise sobre o mercado de coworking brasileiro. Recuperado de https:// coworkingbrasil.org/censo/censo-2015/\#download [14 janeiro 2017]

Eaton, S. C. (2001). If you can use them: flexibility policies, organizational commitment, and perceived productivity. KSG Faculty Research Working Paper Series, 01-009.

Foertsch, C. (2011). The coworker's profile. Deskmag. Recuperado de https://goo.gl/6rKwF [13 janeiro 2011]

Grisci, C. L. I., Bitencourt, B. M., \& Fleck, C. F. (2012). Trabalho imaterial, medo, solidão: "amigos de aluguel” na sociedade líquido-moderna. Psicologia em Estudo, 17 (1), 141-149.

Grisci, C. L. I., Scalco, P. D., \& Kruter, G. E. (2011). Dilemas pessoais no trabalho imaterial bancário. Psicologia $\mathscr{E}$ Sociedade, 23 (3), 564-573.

Jackson, K. (2013). Making spaces for others. 2013. Dissertação de Mestrado. Mestrado em Mídias Digitais, Hyper Island.

Kojo, I. V. I., \& Nenonen, S. (2014). Places for multi-locational work: opportunities for facilities management. Facilities, $33(2), 20-37$.

Mouat, C. M., \& Buksh, B. (2015). Activating smart work hubs for urban revitalisation: evidence and implications of digital urbanism for planning and policy from South-East Queensland. Australian Planner, 52 (1), 16-26.

Pohler, N. (2012). Neue Arbeitsräume für neue Arbeitsformen: coworking spaces. Österreichische Zeitschrift für Soziologie, 37 (1), 65-78.

Half, R. (2012). Coordenar equipe remota é desafio do home-office no Brasil. Recuperado de https://goo.gl/YBSm5U [29 maio 2015]

Spinuzzi, C. (2012). Working alone together: coworking as emergent collaborative activity. Journal of Business and Technical Communication, 26 (4), 399-441.

Uda, T. (2013). What is coworking? A theoretical study on the concept of coworking. Graduate School of Economics \& Business Administration, Hokkaido University. Discussion Paper, Series A, 265, 1-15.

Endereço para correspondência psicopaloma@gmail.com, edite@cfh.ufsc.br
Enviado em: 07/12/2015

Revisado em: 05/12/2016

Aprovado em: 05/12/2016 